A9

doi: 10.14232/ syrpharmacognosy.2021.a9

\title{
Preparation and investigation of ecdysteroid - cinnamic acid hybrid compounds
}

\author{
Márton Háznagy \\ haznagy.marton.benedek@szte.hu
}

During the COVID lock-down, the chemical structures of more than 170 semi-synthetic ecdysteroid derivatives were collected from 21 articles since 2012, when the latest semisynthetic reviews were published. These ecdysteroids, along with the semi-synthetic ecdysteroid library of our group, were processed into SMILES strings and organized according to their available bioactivities. Altogether, more than 300 ecdysteroids and, where available, their biological data were collected into a database in a way that is appropriate for an in-depth in silico evaluation. Further investigations on this database are planned with the ChemGPS-NP [1] software in collaboration.

During the literature survey, some reports on ecdysteroid cinnamic derivatives have been found. These esters were isolated from the fronds of Microsorum membranifolium [2]. This inspired us to prepare related new hybrid compounds through semi-synthesis.

20-Hydroxyecdysone (20E) and cinnamic acid were reacted with $\mathrm{EDCl} . \mathrm{HCl}$ and DMAP in mol. sieve DCM for 4.5 day in room temperature [3]. The crude products were purified via flash chromatography and HPLC. Four ecdysteroid derivatives including two new compounds were obtained, and their structures were confirmed via NMR as the 2-, 2,3-, 2,22, and the 3,22cinnamates of $20 \mathrm{E}$. The compounds exhibited moderate antioxidant activities on DPPH and ORAC assays. Synthesis of the caffeate and ferulate derivatives of $20 \mathrm{E}$ are currently ongoing.

Supervisors: Attila Hunyadi, Zsolt Szakonyi

\section{Acknowledgements:}

This work was supported by the National Research, Development and Innovation Office, Hungary (NKFIH; K-134704), the Economic Development and Innovation Operative Program GINOP-2.3.2-15-2016-00012, and the Ministry of Human Capacities, Hungary grant 20391-3/2018/FEKUSTRAT and the EFOP 3.6.3-VEKOP-16-2017-00009 project.

\section{References:}

[1] Larsson J, et al. Journal of Natural Products 2007; 70(5):789-794.

[2] Ho R, et al. Molecules 2012; 17(10):11598-11606.

[3] Xu S, et al. Fitoterapia 2014; 99:300-306. 